Relations industrielles

Industrial Relations

\title{
Nouvelle présence de l'État dans les relations du travail
}

\section{Fernand Morin}

Volume 39, numéro 4, 1984

URI : https://id.erudit.org/iderudit/050081ar

DOI : https://doi.org/10.7202/050081ar

Aller au sommaire du numéro

\section{Éditeur(s)}

Département des relations industrielles de l'Université Laval

\section{ISSN}

0034-379X (imprimé)

1703-8138 (numérique)

Découvrir la revue

\section{Citer cet article}

Morin, F. (1984). Nouvelle présence de l'État dans les relations du travail. Relations industrielles / Industrial Relations, 39(4), 744-752.

https://doi.org/10.7202/050081ar
Résumé de l'article

Nouvelle presence de l'Etat dans les relations de travail
Tous droits réservés @ Département des relations industrielles de l'Université Laval, 1984
Ce document est protégé par la loi sur le droit d'auteur. L’utilisation des services d'Érudit (y compris la reproduction) est assujettie à sa politique d'utilisation que vous pouvez consulter en ligne.

https://apropos.erudit.org/fr/usagers/politique-dutilisation/ 


\section{Nouvelle présence de l'État dans les relations du travail}

\section{Fernand Morin}

Le droit du travail et plus particulièrement le droit des rapports collectifs du travail évolue constamment dans le but de suivre le mouvement des faits et de tenter de s'y adapter. Ces essais d'adaptation ne sont certes pas toujours réussis et peuvent produire des effets insoupçonnés. Si cette évolution est parfois accélérée, en d'autres cas, elle peut être retardée ou arrêtée selon les contingences: marché du travail, situation économique générale, force ou faiblesse des parties en présence, rôle plus ou moins dynamique du gouvernement en place, etc. Aussi, est-il opportun de s'interroger sur le rôle de l'État dans les relations du travail en 1984 et, d'une façon plus directe, d'entrevoir ses implications pratiques immédiates et à moyen terme sur l'administration des entreprises.

À ces fins, nous proposons d'étudier certains changements apportés au Code du travail pour en signaler, par la suite, les effets et la portée sur la gestion des entreprises. Il va sans dire que cet exposé ne pouvait être exhaustif; bien plus, il est délibérément partiel. Nous n'y traitons que de l'évolution du Code du travail et nous ne soulevons que quelques éléments de la Loi sur les normes du travail et de la Loi sur la santé et sécurité au travail.

Cette analyse des récents changements apportés au régime des relations du travail n'a pas 'pour but premier de contester directement la valeur respective de chacune de ces nouvelles règles de vie en milieu de travail. Pour la plupart, en considérant leur objet et leur finalité respectives, il nous serait d'ailleurs difficile d'en contester la valeur ou de ne pas reconnaître leur opportunité. Nous essaierons simplement de considérer les implications générales résultant de leur inévitable conjugaison.

On ne peut étudier les changements apportés au Code du travail sans d'abord rappeler les règles fondamentales de cette loi. Le Code du travail de 1964 remettait à jour la Loi des relations du travail de 1944 et cette dernière, on le sait, était élaborée selon le modèle américain. Or, ni en 1944 et ni en 1964 , les trois principes fondamentaux de ce régime des rapports collectifs déjà retenus aux Etats-Unis en 1935 pour édicter le Warner Act n'ont été remise en cause. Ces trois règles principales sont:

i) L'entreprise ou une de ses parties à titre de cadre exclusif pour le tenue de ses rapports collectifs: il s'agissait alors d'un choix délibéré puisque les régimes européens alors connus étaient aménagés sur une base sectorielle ou intersectorielle, nationale ou régionale

- MORIN, F., professeur, Département des relations industrielles, Université Laval. 
ii) la présence exclusive d'un seul syndicat à la fois: ce monopole de représentation consacré par l'accréditation devait faciliter et simplifier la tenue de rapports collectifs au lieu d'imposer à l'employeur plusieurs vis-à-vis;

iii) les rôles de modérateur, pondérateur, pacificateur et d'intendant étaient réservés à l'État. La négociation devait demeurer l'affaire exclusive des parties, c'est dire qu'elle restait du domaine privé: l'État ne devait intervenir que pour baliser la voie et maintenir à un niveau tolérable l'usage respectif des moyens de pression.

Il y a donc trois unités: un seul syndicat, une seule entreprise et une seule convention collective. En somme, on transposa, au plan collectif, la règle sacro-sainte de l'autonomie des volontés des personnes, si chère aux civilistes.

Au sujet de la première règle, celle du cadre de la négociation collective limitée à l'entreprise, elle n'est toujours pas modifiée bien qu'elle soit remise en cause: ce débat s'effectue sous le thème de la négociation sectorielle. Par ailleurs, les deux autres principes concernant le rôle du syndicat et celui de l'État furent modifiés ou limités en multiples occasions. En quoi ou comment ces deux règles furent-elles modifiées et quels sont les effets que l'on peut déjà en dégager?

Les ajouts de 1978 et 1982 apportés au Code du travail peuvent être rangés en trois sous-groupes: les premiers traitent du rôle de l'État; les deuxièmes, de la relation syndicat-salarié et les troisièmes, du statut de l'Employeur et de ses prérogatives. Un bref rappel de ces règles nouvelles permet néanmoins de voir comment ces modifications sont interreliées et quels sont les effets de leur inévitable conjugaison.

\section{LE NOUVEAU RÔLE DE L'ÉTAT POUR LA TENUE DE RAPPORTS COLLECTIFS}

La puissance publique peut maintenant intervenir dans les rapports collectifs sous de multiples formes et en maintes occasions. Ce droit d'intervention est relativement bien articulé à la loi constitutive du ministère du Travail où on retrouve les articles 15 et 16 qui se lisent ainsi:

«En tout temps, le ministre peut désigner une personne pour favoriser l'établissement ou le maintien de bonnes relations entre un employeur et ses salaries ou l'association qui les représente. Cette personne fait rapport au ministre du demande."

«Dans l'exercice de ses fonctions, le ministre peut, par lui-même ou une personne qu'il désigne, enquêter sur toute matière de sa compétence.»

Cette nouvelle volonté d'intervention ou de participation directe se manifeste de multiples manières et notamment:

- en obligeant les parties à donner au ministre copie de tout avis de négociation: il lui est ainsi possible de suivre de près toute négociation collective (art. 52.1) et d'intervenir au besoin; 
- le ministre peut maintenant dépêcher un conciliateur auprès des parties et ce, en tout temps au cours de la négociation et de sa seule autorité (art. 55). Autrefois, les parties devaient demander cette intervention et la conciliation avait trop souvent une vocation stratégique;

- dans le cas d'une première convention collective, le ministre peut nommer un arbitre chargé de régler le différend c'est-à-dire d'édicter les conditions de travail au lieu et place des parties (art. 93.1 et ss.);

- un enquêteur du ministre peut être chargé de vérifier si les parties respectent les articles, art. 109.1 et ss. relatifs à la protection de l'exercice du droit de grève (les briseurs de grève). Autrefois, le ministère ne s'approchait que très rarement des lignes de piquets;

- le ministre peut charger un enquêteur d'intervenir lorsqu'un salarié n'est pas satisfait des services du syndicat à la suite de son renvoi ou d'une mesure disciplinaire $(47.3 \mathrm{Ct})$ ou encore, de son non-rappel au travail à la suite d'une grève ou d'un lock-out (110.1 Ct);

- le ministre doit être informé de toute décision autorisant le syndicat à faire grève et dans les quarante-huit (48) heures qui suivent une grève ou un lock-out l'initiateur doit en informer le ministre (20.2 et 58.1).

- le choix du mécanisme d'arbitrage relève de moins en moins des parties: il n'y a plus de conseils ni de tribunaux d'arbitrage mais des arbitres (art. 77, 93.3 et 100.1.1 Ct) et les parties ne peuvent que désigner des assesseurs qui ne participent pas à la prise de décision (art. 88 et $100.11 \mathrm{Ct}$ ).

Ces sept cas illustrent bien ce nouveau rôle du ministre du Travail, sa nouvelle vocation interventionniste soit directement, soit par personne interposée simple ou double (enquêteur, conciliateur, médiateur, etc.). Par ces nouveaux services de recherche et d'information et par la voie de la conciliation préventive, le ministère du Travail sera ou pourra être présent à toutes les phases de la négociation collective, de son propre chef et non seulement, sur invitation stratégique ou en situation catastrophique. Outre cette présence de l'État à la table de négociation devenue, en quelque sorte, triangulaire, nous pourrons constater que ces interventions s'effectuent aussi en amont et en aval du processus même de la négociation.

\section{INTERVENTION DE L'ETAT DANS LE RAPPORT SYNDICAT/SALARIÉ}

Il s'agit manifestement de l'intervention la plus importante et pouvant avoir des effets encore difficiles à évaluer. Jusqu'au 22 décembre 1977, le législateur s'était abstenu d'intervenir d'une façon directe dans les affaires du gouvernement syndical. À titre de représentant exclusif des salariés, le syndicat accrédité devait exprimer la volonté collective du groupe auprès de l'employeur sans que l'on puisse, avant comme après, contrôler la qualité de son mandat. Les interventions connues jusqu'alors s'effectuaient par le biais des tribunaux judiciaires et se fondaient sur les principes fondamentaux de justice qui avaient pu être bafoués ou déclarés tels. Il en est maintenant autrement et cela, au sujet de trois questions importantes: 
La contribution obligatoire des salariés aux frais du syndicat

Selon l'art. 47 du Code du travail, dès qu'un syndicat est accrédité, l'employeur doit retenir sur la paie de chaque salarié visé un montant égal à la cotisation syndicale et le remettre au syndicat. Cette première disposition complétée par l'art. 63 reconnaissant, d'une certaine manière, la légalité des clauses de sécurité syndicale (l'obligation conventionnelle d'adhérer au syndicat accrédité) mettent ainsi fin à un long débat judiciaire. De plus, ces mêmes questions faisaient souvent l'objet de longues et difficiles négociations et ce, jusqu'à ce 22 novembre 1977 . Maintenant que l'État a tranché, la discussion à la table de négociation à ce sujet en est fort abrégée.

Cette intervention législative a pour premier effet d'assurer au syndicat accrédité une source de financement régulière et certaine. Il est encore bien difficile de préciser les implications pratiques de ces nouvelles règles qui peuvent changer la nature même de la relation entre un salarié et le syndicat qui le représente vis-à-vis son employeur. Peu importe dorénavant si ce salarié voulait se syndiquer, sans égard à sa volonté à ce sujet, il se doit de contribuer aux frais engagés par le syndicat accrédité. Ce dernier peut, peu importe sa popularité du moins pour un temps, être assuré de la contribution financière de tous les salariés visés à l'unité de négociation. Dans quelle mesure un tel changement peut-il modifier le comportement des élus syndicaux? Comment un salarié ainsi forcé de contribuer percevra-t-il ce syndicat? En plusieurs cas, un tel syndicat pourrait être perçu comme une simple association de services où la question de la solidarité serait rangée au niveau du folklore, où l'adhésion syndicale s'assimilerait à une prise d'une police d'assurance quasi-obligatoire. En somme, l'attente d'un tel syndiqué pourrait être semblable à celle d'un automobiliste face à son club automobile, etc. Cette désaffectation, ce repli égoiste peut cependant provoquer des contrecoups et, comme nous le verrons, même à l'égard de l'employeur. Si, par le concours d'une loi, le support financier est assuré au syndicat, c'est que ses créneaux sont aussi modifiés: il devient de plus en plus une institution publique.

\section{La démocratie syndicale par édit}

Depuis ces amendements de 1977-1978, tout syndicat accrédité doit suivre une procédure précise pour être autorisé à faire grève ou pour conclure une convention collective (art. 20.1 et $20.2 \mathrm{Ct}$ ). Parce que certains dirigeants syndicaux avaient abusé de leur pouvoir et de leur position et négligé parfois de consulter leurs membres, le législateur a imposé une même formule décisionnelle pour tous. Dorénavant, les syndiqués visés doivent préalablement autoriser le syndicat par voie d'un scrutin secret pour faire grève ou conclure une convention collective. Déjà, on pourrait poser certaines questions à l'égard des effets d'une telle conception de la démocratie syndicale:

- un tel vote, imposé par la loi et effectué au su et au vu de tous, ne devient-il pas un vote de confiance en faveur des dirigeants et ainsi, ne change-t-il pas de nature? 
- un tel vote ne peut-il pas prendre parfois l'allure d'un excellent moyen stratégique pour convaincre l'employeur et le public du sérieux des demandes syndicales et de leur détermination à prendre les moyens pour les obtenir alors que la volonté collective n'est pas réellement à ce point arrêtée?

- si une minorité active du syndicat et concentré en des services-clés de l'entreprise décide de faire grève, que peut faire le syndicat qui s'abstient puisque la majorité en aurait décider ainsi? Pourquoi ce syndicat serait-il responsable des actes d'un sous-groupe dissident alors que ce syndicat respecte la consigne donnée légalement par la majorité des membres?

- si la majorité des syndiqués refuse d'autoriser le syndicat à conclure une convention collective alors que son comité de négociation l'aurait préalablement acceptée, que peut faire l'employeur? Les dirigeants syndicaux peuvent-ils convoquer un second scrutin sans ajout de la part de l'employeur?

Ces quelques exemples visent seulement à illustrer que la question de la démocratie syndicale dépasse largement le simple cadre de la mécanique (art. 20.2 et 20.3 C.T.) et qu'à ne vouloir s'en tenir à cette dimension superficielle, les effets des solutions retenues peuvent parfois être pire que la situation que l'on entendait corriger. Ainsi, ceux qui croyaient que ces ajouts de procédure à facture démocratique pouvaient alourdir le processus décisionnel syndical et réduire le nombre de grèves, pourraient, au cours des prochaines années, avoir quelques surprises.

\section{Les services de représentation que doit fournir un syndicat}

En deux cas précis, celui du renvoi ou d'une mesure disciplinaire et celui du refus de l'employeur de reprendre un salarié à la suite d'une grève ou d'un lock-out, un salarié peut demander un contrôle des décisions syndicales qui s'en suivent et, sur autorisation du tribunal du travail, imposer l'arbitrage. Ainsi, au-delà des règles de procédure de la convention collective et de l'extinction des délais conventionnels, tout règlement conclu entre le syndicat et l'employeur, en ces cas, pourrait alors être remis en cause. La portée de ce nouveau recours dépend, bien sûr, de l'approche du tribunal du travail appelé à autoriser un tel recours spécial. C'est dire qu'en ces matières, une bonne décision syndicale ou un bon accord entre un employeur et le syndicat peuvent dépendre de la conception que s'en fera le tribunal. En d'autres cas, on peut fort bien imaginer la connivence même du syndicat qui peut, en raison de la prescription du moyen direct ou pour d'autres fins stratégiques, encourager le syndiqué à exercer lui-même ce recours par la voie du tribunal du travail. Comment alors l'employeur peutil être assuré de la valeur des ententes qu'il peut conclure à ces mêmes sujets avec le syndicat accrédité? Par le truchement du tribunal du travail, ces règlements ou accords explicites ou tacites peuvent être remis en cause. N'est-ce pas là une autre façon de constater que les rapports collectifs du travail ne sont plus l'affaire exclusive de l'employeur et du syndicat accrédité? 


\section{MODIFICATION DU STATUT ET DES POUVOIRS DE L'EMPLOYEUR}

La présence plus active de l'Etat dans les rapports collectifs du travail, soit par des interventions directes, soit par des modifications au Code du travail, pour préciser les rapports des salariés et des syndicats accrédités ont des effets certains sur le rôle et la liberté de manoeuvre de l'employeur. De plus, la législation du travail de 1977 et 1982 modifia également certaines prérogatives de l'employeur: ces changements sont parfois directs et immédiats alors que dans d'autres cas, il faudra encore quelques années avant d'en percevoir les effets réels. À simple titre d'illustration et sans vouloir être exhaustif, nous soulignons quelques changements apportés au statut et aux pouvoirs de l'employeur pour l'aménagement des conditions de travail.

\section{Questions mises hors du champs de la négociation}

Jusqu'à tout récemment encore, les parties jouissaient d'une pleine liberté de convention. Ne déclare-t-on pas à l'article 62 du Code du travail que seules les questions contraires à l'ordre public et aux lois ne peuvent faire l'objet de la négociation collective? Cette grande liberté de manoeuvre permettait aux parties d'adapter les conditions de travail aux contraintes de chaque milieu et à leurs contingences propres. Puisque l'employeur et le syndicat sont obligés de négocier de bonne foi, sans toutefois être tendu de conclure une convention collective sur des points précis (art. $53 \mathrm{Ct}$ ), cette même liberté pouvait permettre à l'employeur de refuser de négocier certains points pour des raisons de «principe», politiques ou économiques, etc. Maintenant, le législateur a retiré quelques-unes de ces mêmes questions épineuses de l'ordre du jour de la table de négociation: l'Etat impose d'autorité des règles sur ces mêmes points en remplacement d'autant de dispositions des conventions collectives qui pouvaient en traiter (du moins pour leur partie contraire ou inférieure). Cette technique facilite la protection qu'entend alors conférer l'État aux salariés sans qu'il puisse y avoir, au préalable, négociation, tractation, compromis ou marchandage à ces sujets ou à ces occasions.

Parmi les questions ainsi soutirées de la négociation ou de l'initiative des parties, mentionnons:

- celle du précompte généralisé: tous les salariés doivent contribuer aux frais du syndicat accrédité (l'équivalent de la cotisation); ces sommes sont retenues à la source par l'employeur (art. 47 C.T.);

- celle de la langue des relations du travail: les communications au personnel, la convention collective et les sentences arbitrales doivent être rédigées en français (art. 41, 43 et 44 - Charte de la langue française): autrefois, l'ancien article 51 C.T. laissait aux parties le soin de négocier cette question;

- celle relative au droit du salarié de recouvrer prioritairement son emploi au lendemain d'une grève ou d'un lock-out (art. 110.1 C.T.);

- celle du droit à l'arbitrage pour tout salarié ayant 5 ans de service qui croit avoir été injustement congédié (art. 124 L.N.T.), sans égard à la présence d'un syndicat. (Ce recours s'applique à tous les cas non régis par une convention collective garantissant un contrôle équivalent.) 
- celle de la fin de la négociation, dans le cas d'une première expérience: les conditions de travail peuvent être imposées par décision d'un arbitre, selon les prescriptions établies aux articles 93.1 C.T.

\section{Élévation du seuil de la négociation}

En plusieurs autres domaines, le législateur a établi des normes minimales que les parties doivent respecter. S'il leur est possible de les adapter et même de les compléter lors de la négociation de la convention collective, elles ne peuvent, directement ou indirectement, établir des conditions de travail contraires, moins avantageuses ou incompatibles. Ces normes minimales servent dorénavant de base ou de point de départ à toute négociation collective et elles comprennent notamment ces éléments:

- la procédure arbitrale aménagée au Code du travail en 29 dispositions sert de solide encadrement à cette «justice parallèle» destinée à contrôler efficacement l'application de la convention collective. En pratique, cela signifie que ce sont les décisions de l'employeur qui sont généralement soumis à ce contrôle puisque c'est lui qui prend la plupart des initiatives en ce domaine (paie, surtemps, promotion, mesures disciplinaires, etc.);

- le pouvoir de rescinder les mesures disciplinaires de l'employeur par voie d'arbitrage y compris la réintégration du salarié à son poste: seuls les cas spécifiés à la convention collective peuvent être soustraits à ce pouvoir (art. 100.12 C.T.). En inversant ainsi la règle (auparavant, les parties devaient conférer à l'arbitre un tel pouvoir), le rôle des parties à la négociation est aussi modifié: c'est l'employeur qui doit chercher à obtenir quelques accommodements alors que le syndicat n'a qu'à s'abstenir, s'il entend laisser à l'arbitre un complet contrôle en cette matière;

- les conditions de travail édictées par la Loi sur les normes du travail ou par règlements émis en vertu de cette même loi s'imposent à tous y compris aux parties liées par convention collective. Parmi les normes de travail que renferme cette loi et qui seront complétées par règlement (en sus ou en moins) nous pouvons rappeler les suivantes: le salaire minimum; la base de 44 heures pour établir la paie supplémentaire (art. 55 L.N.T.); le minimum de 7 jours fériés et payés (art. 60 L.N.T.); le congé annuel de 2 semaines après une année de service, de 3 semaines, après 10 ans de service (art. 68 L.N.T.); certains congés sociaux (décès, mariage, naissance) (art. 80 L.N.T.); le préavis de fin d'emploi: une semaine pour tout salarié ayant 3 mois de service et moins de 12 mois, 4 semaines, pour tout salarié de 5 ans de service à 10 ans et de 8 semaines, pour tout salarié de plus de 10 ans; à son départ, tout salarié peut exiger un certificat de travail (art. 84 L.N.T.); le congé de maternité (art. 89 al. 6 et 157 L.N.T.), etc. Cet ensemble de normes du travail (non limitatif) s'impose aux parties liées par une convention collective: elles peuvent les compléter, les adapter, mais elles ne peuvent s'entendre pour s'y soustraire (art. 93 L.N.T.). Le plus important n'est peut-être pas les conditions de travail ainsi édictées hors de l'entreprise et de la table de négociation, mais les occasions que cette loi procure pour élever le niveau de la négociation et pour l'orienter. 
$\mathrm{Au}$ sujet de la Loi sur les normes du travail, l'élément le plus caractéristique, et encore le plus difficile à cerner pour l'instant, consiste en la remise d'un pouvoir réglementaire important au gouvernement. Les modalités d'application et les règles complémentaires à ces normes de travail doivent être édictées par voie de règlement arrêté directement par le Conseil des ministres (art. 88 L.N.T.). On peut croire que l'intensité de cette intervention étatique variera selon les partis au pouvoir et d'une façon plus immédiate, selon la personnalité du ministre en fonction. Ainsi, compte tenu des implications de ces normes sur la négociation collective, le lobbying patronal et syndical s'accentuera sans aucun doute. Aussi contradictoire que cela puisse paraître, les parties susciteront encore l'intervention de l'État mais, il est vrai, pour des fins respectivement opposées.

La Loi sur la santé et la sécurité au travail établit également un régime nouveau auquel est soumis l'entreprise: les conventions collectives peuvent compléter ces règles (art. 4 L.S.S.T.). Cette loi, on le sait, établit clairement que les contraintes de production et les objectifs de l'entreprise ne peuvent prévaloir sur la santé et la sécurité des salariés et que ces derniers n'ont plus à s'en remettre à la seule vigilance de l'employeur pour leur protection. Pour l'instant, nous nous limitons à ne rappeler que ces éléments caractéristiques de cette loi:

- l'article 12 précisant les modalités d'exercice du droit de refuser de travailler si le salarié croit que sa santé ou sa sécurité ou celle de ces collègues est mise en danger;

- les articles 32 et ss. établissant le retrait préventif du salarié de certains postes si son état de santé l'exige;

- l'article 52 imposant à l'employeur la tenue d'un registre des caractéristiques de chaque poste de travail (cette opération devrait l'obliger à prendre conscience de la gravité des risques inhérents à chaque tâche et par la suite, on ne pourra croire qu'il les ignorait);

- l'article 58 demandant que l'employeur élabore un projet de programme de prévention susceptible d'éliminer les sources de dangers et le soumettre pour avis au comité paritaire de santé et de sécurité et le faire approuver par la Commission;

- l'article 68 prévoyant que les établissements ayant plus de 20 salariés doivent constituer des comités paritaires de santé et de sécurité au travail qui choisissent le médecin responsable des services de santé et surveillent l'application du programme de prévention;

- selon l'article 186, les inspecteurs de la Commission pourront suspendre l'exécution de travaux ou imposer provisoirement la fermeture d'un lieu de travail s'ils le jugent nécessaire à la protection de la santé et de la sécurité des travailleurs.

Il nous faut donc constater que les questions relatives à la santé et à la sécurité des travailleurs ne ressortent plus du seul cadre de la responsabilité civile de l'employeur à titre de propriétaire des moyens de production. On ne peut plus aborder ces questions sous l'angle des obligations du «bon père de famille" que devait être l'employeur selon le modèle civiliste. Les travailleurs, directement et par le truchement de leur syndicat accrédité, ont 
dorénavant des droits bien établis en cette matière et pourront participer activement et de leur propre chef à la gestion de ces dossiers. Il apparaît alors évident que ces éléments nouveaux constituent une autre facette modifiant la gestion de l'entreprise.

Ce simple rappel de quelques changements apportés au régime des relations du travail au Québec permet de constater, à tout le moins, que l'entreprise et le syndicat ne peuvent plus être considérés comme des organisations du seul domaine privé. Trop d'éléments extérieurs participent à leur opération et à leur gouvernement pour que l'on puisse continuer de ramener ou de réduire l'entreprise à la simple notion de la propriété privée de moyens de production et le syndicat, à une association privée. Sans considérer les questions d'ordre fiscal, ni le support de l'État, en amont et en aval, aux multiples étapes du processus de production et de distribution des entreprises et à leur financement, ne peut-on arriver aussi à ces mêmes constats en considérant la seule question des relations du travail? En effet,

- par les normes du travail servant d'assise à toute relation de travail;

- par l'intervention de l'État ou par sa présence presque continue aux rapports collectifs du travail;

- par le mécanisme de contrôle des décisions de l'employeur concernant l'administration des conventions collectives et des mesures disciplinaires;

- par l'encadrement imposé à la gestion syndicale;

- par le rôle direct des salariés et de leurs représentants qui leur est maintenant reconnu pour la protection de leur santé et de leur sécurité

la gestion de l'entreprise et du syndicat subit une métamorphose certaine, lente et continue et qui devrait imposer des changements d'attitudes, d'optiques et de comportement de la part des gestionnaires et de leurs conseillers. D'autre part, il serait inexact de croire que cette "publicisation» de l'entreprise exigerait moins d'initiative, moins d'entrepreneurship qu'autrefois de la part des administrateurs. Les défis sont certes différents, mais toujours présents et peut-être, sont-ils plus complexes et moins spectaculaires qu'autrefois. Si cette évolution exige une réforme de la part des administrateurs d'entreprise, on ne peut ignorer qu'une pareille remise en cause s'imposera aussi aux dirigeants du mouvement syndical. Le rôle que l'État leur confie et les services que les salariés peuvent exiger d'eux sont également bien différents de ceux qui leur étaient dévolus à l'époque de la simple revendication. Le comportement et l'approche des gestionnaires syndicaux devraient également se modifier. En d'autres termes, la socialisation progressive de l'entreprise exigera une approche fort différente de la part de tout gestionnaire. 